\title{
Use of glucocorticoids and azithromycin in the therapy of COVID-19
}

\author{
Miguel de Lemos Neto $^{1} \cdot$ Rafael Costa Vieira Alexandre ${ }^{2} \cdot$ Rafaela Oliveira Gallart Morra ${ }^{2}$. \\ Juliana Aparecida Souza da Paz ${ }^{3}$. Shana Priscila Coutinho Barroso ${ }^{3}$. Angela Castro Resende ${ }^{4}$. \\ Daniel J. M. de Medeiros-Lima' ${ }^{10}$. Pedro Celso Braga Alexandre ${ }^{2}$
}

Received: 26 April 2021 / Revised: 23 May 2021 / Accepted: 25 May 2021 / Published online: 4 June 2021

(c) Maj Institute of Pharmacology Polish Academy of Sciences 2021

\begin{abstract}
In December 2019, a new variant coronavirus, SARS-CoV-2, emerged in China, which was initially described as a pneumonia of an unknown agent. The new coronavirus spreads mainly by person-to-person transmission through close contact. The pathophysiology of COVID-19 is related to a complex immune system response that varies between people and, in severe cases of the disease, is characterized by excessive responses called "cytokine storms," which are associated with complications that can lead to a state of hypercoagulation and death. Glucocorticoids and azithromycin are drugs that may be effective in the treatment. This review aims to highlight the clinical findings that demonstrate the effectiveness of glucocorticoid and azithromycin therapy in the treatment of COVID-19. To date, many drugs have been studied for use in combination therapy, and the rapid expansion of knowledge about the virology of SARS-CoV-2 generates a more accurate direction in therapy.
\end{abstract}

Keywords COVID-19 $\cdot$ Glucocorticoids $\cdot$ Antibiotics $\cdot$ SARS-CoV-2

\section{Introduction}

In December 2019, cases of pneumonia were reported in Wuhan city, China. A novel coronavirus (SARS-CoV-2) was found to be the causative agent of the illnesses. On February 11, 2020, the disease was named COVID-19 by the World Health Organization (WHO) [1, 2]. SARS-CoV-2 is a variant of the coronavirus $(\mathrm{CoV})$, a family of viruses known in the 1960s, which received its name due to the spicules on its surface that resemble a crown (in Latin-corona). Coronaviruses (CoVs) are classified within the family Coronaviridae, subfamily Coronaviriae and comprise four genera: alpha $(\alpha)$, beta $(\beta)$, gamma $(\gamma)$, and delta ( $\delta)$ [3]. SARS-CoV-2 has been classified as a new member of the genus $\beta$ coronavirus

Daniel J. M. de Medeiros-Lima

daniel.jose@fmc.br

1 Faculty of Medicine of Campos dos Goytacazes, Av. Alberto Torres, 217, Campos dos Goytacazes, Brazil

2 Department of Pharmacology, Federal University of the State of Rio de Janeiro, Rio de Janeiro, Brazil

3 Molecular Biology Laboratory, Biomedical Research Institute, Marcílio Dias Navy Hospital, Rio de Janeiro, Brazil

4 Department of Pharmacology, Institute of Biology, Rio de Janeiro State University, Rio de Janeiro, Brazil by sequencing its genome and presents as a positive-sense, single-stranded RNA virus of approximately $27-32 \mathrm{~kb}$ [4]. Its genome is made up of four major structural proteins: the transmembrane spike (S) and envelope (E), the latter in lesser quantities. It also has the more abundant membrane protein $(\mathrm{M})$, which supports the envelope, and nucleocapsid $(\mathrm{N})$, formed by phosphoprotein $\mathrm{N}$, which binds to the viral RNA. All are encoded at the 3 ' end of the genome $[5,6]$

Coronavirus infections can cause various health conditions, ranging from common cold to severe respiratory syndromes, such as severe acute respiratory syndrome (SARS-CoV) and the Middle East respiratory syndrome (MERS-CoV) [1]. SARS-CoV was responsible for an outbreak in 2002, which started in China and spread to Southwest Asia, North America, Europe, and South Africa. This outbreak reached pandemic proportions and respiratory syndrome pictures were observed, with a mortality rate close to 9.6\%. In 2012, MERS-CoV became responsible for severe respiratory syndrome in the Middle East and South Korea, with cases exported in other countries, with an approximate $35 \%$ mortality level [7].

The urgency and emergency of treatment for patients affected by COVID-19 generated a search for drugs that could be used in the treatment of this disease. Concomitantly, there is the impossibility to plan and implementing 
works that generate robust scientific evidence on the use of certain drugs in a short time. Therefore, many researchers look for the repositioning of drugs already approved to control the infection by the virus [8,9]. Drugs already used for other purposes have been shown to exert an antiviral effect through various mechanisms, including blocking viral entry into the cell, inhibiting an enzyme encoded by the virus, or blocking particle formation [9]. Among the agents used in Brazil to control infection by COVID-19, the corticosteroids and azithromycin stand out.

Corticosteroids used as therapeutic agents have antiinflammatory and immunomodulatory properties. The mechanisms of action are characterized by the activation or inhibition of various target genes. These molecules act on many cells involved in innate immunity (macrophages, granulocytes, mast cells) and adaptive immunity (lymphocytes). They also act on other cells (fibroblasts, epithelial and endothelial cells). The anti-inflammatory efficacy can be explained by inhibition of the synthesis of numerous cytokines, enzymes and, mediators of inflammation or induction of cytokines and anti-inflammatory molecules [10].

Azithromycin is an azalide, a subclass of the macrolide antibiotics. It is derived from erythromycin, with a nitrogen atom substituted with methyl incorporated in the lactone ring, making the 15-molecule lactone ring. It prevents bacteria from growing by interfering with the synthesis of their proteins. Because it binds to the 50S subunit of the bacterial ribosome, it promotes inhibition of mRNA translation $[11,12]$.

The purpose of this review is to evoke evidence on the use of corticoids and azithromycin as a proposed treatment in the therapeutic intervention for COVID-19, in addition to compiling data on the pathophysiology of the disease.

\section{Methodology}

This work has a qualitative approach with an exploratory purpose. Bibliographic research was used as a means to carry out this review. All the exposed content was based on bibliographic references found in scientific publication media. The articles made available by the WHO for guidance and directives on COVID-19 were also used as reference material. The databases used for this work were PubMed, Scielo, LILACS, Capes, and Google Scholar.

The following terms were used to perform the searches: Coronavirus, Severe Acute Respiratory Syndrome and coronavirus, 2019-nCoV, SARS-CoV-2, SARS-CoV, MERS-CoV, COVID-19, SARS-CoV and Pathophysiology, SARS-CoV and Inflammation, SARS-CoV and Corticoids, SARS-CoV and Azithromycin. All materials used for consultation were published from January 1, 2020, to January 1, 2021.

Approximately 824 articles were found on the keywords addressed. To carry out this work, 50 articles were chosen. The criterion for choosing was the relevance and number of citations obtained from each work.

\section{Pathophysiology}

SARS-CoV-2 is transmitted predominantly by respiratory tract secretions through drops and potentially by the fecal-oral route, conjunctiva, and fomites. Fluids such as respiratory tract droplets, saliva, feces, and urine can be used to trace the path of transmission of viral infection [13]. Primary replication presumably occurs in epithelial mucosa of the upper respiratory system (pharyngeal nasal cavity), then spreads to the lower respiratory system and gastrointestinal mucosa, leading to viremia with a medium level of viral load. At this stage, in a certain number of patients, the infection remains controlled and asymptomatic. In other patients, non-respiratory conditions are observed with acute liver and heart damage, renal failure, and diarrhea $[14,15]$.

Angiotensin-converting enzyme (ACE2) is a functional receptor used by SARS-CoV-2 to enter the cell. This type I membrane protein is expressed in the lung, heart, kidney, and intestine and is mainly associated with cardiovascular disease [16-18]. Coronavirus infection is initiated by the interaction of the viral envelope with the host's cell membrane. The virus can bind to the ACE2 protein through its Spike $\mathrm{S}$ protein. This binding promotes conformational changes in one of its subunits (S1), promoting a fusion (subunits S2) of the viral envelope in the cell membrane and subsequently the endocytosis of the viral genetic material $[16,17,19]$. This process is operated by a type of protease serine TMPRSS2 (receptor transmembrane protease serine 2 ), which has a key role in the cleavage between the $S 1$ and $\mathrm{S} 2$ units. The remaining viral S2 unit drives and completes the fusion in the host's cell membrane [19].

SARS-CoV-2 exhibits a similar response to that exhibited in SARS-CoV infection, but there is biophysical and structural evidence to suggest that SARS-CoV-2 probably binds to human ACE2 with 10-20 times more affinity than SARS-CoV [14]. Protein S from both strains of the virus can down-regulate ACE2 and induce the release of the catalytically active ectodomain of ACE2. ACE2 has a pivotal role in metabolizing angiotensin II to angiotensin [1-7], which in turn reduces adverse reactions that include endothelial dysfunction, enhanced inflammation state, and increased coagulation [19]. Indeed, SARS-CoV-2 can increase inflammation and vascular permeability by raising the levels of angiotensin II. This event can cause severe cell death and 
severely damage vital organs such as the lung, heart, and kidneys $[5,17]$.

The infection promoted by the virus induces an excessive immune reaction in the host, which in turn generates a "cytokine storm," composed of pro-inflammatory cytokines that act on cardiovascular cells, as well as in secondary hemophagocytic lymphohistiocytosis [20]. Patients with severe COVID-19 infection tend to have much higher plasma levels of the cytokines IL-6, IL-8, IL-10, and TNF- $\alpha$ than patients without severity. Also, IL-6 and IL-10 levels show a continuous increase in severe patients. The progression of COVID-19 can be predicted from the analysis of cytokine levels. Among these, interleukin 6 (IL-6) plays a central role in the "cytokine storm". It is produced mainly by macrophages and activated leukocytes and is responsible for activating and differentiating $\mathrm{B}$ and $\mathrm{T}$ lymphocytes, for inducing the production of acute phase proteins, such as C-reactive protein. In addition to inducing fever. It can also suppress the functions of T lymphocytes, dendritic cells, and macrophages that aim to eliminate the coronavirus, which decreases the ability of the immune system to eliminate the infection $[3,21]$. The "cytokine storm" can promote severe damage to vital organs, inducing multiple organ failure, sepsis, myocardial and vascular injury. Concerning the heart for example cytokines can depress myocardial function immediately by activating the neural and subacute sphingomyelinase pathway via beta-adrenergic dullness mediated by nitric oxide signaling. Through evaluations carried out by polymerase chain reactions (RT-PCR), the presence of viral particle was verified in cardiomyocytes, which points to evidence of toxicity in this organ [19].

Uncontrolled lung inflammation is probably the main cause of fatality in SARS-CoV-2 infection. However, the reason for these inflammatory processes in response to SARS-CoV-2 infection has not yet been fully elucidated [5]. The virus initiates its replication by entering the lung target cells (pneumocytes II) through interaction with the ACE2 receptor. This triggers a complex immune response that occurs through the activation of pathogenic Th1 cells, CD14+, CD16+, monocytes, alveolar macrophages, and Th17 lymphocytes. From this, large amounts of cytokines and chemokines are released which attract more inflammatory cells to migrate to the site of inflammation from the blood vessels, thus amplifying the inflammatory response. This inflammatory response is characterized by lung infiltration with neutrophils and macrophages, reactive pneumocyte hyperplasia, thickening of the alveolar walls, and the presence of exudate [5].

As a result of all this inflammatory process, the alveolar epithelial cells become damaged and the extensive secretion and exudation of phlegm cause significant inhibition of the ventilation function of the lungs, leading to hypoxemia, hypotension, and even shock. This factor results in an inefficient supply of oxygen to various organs, including the heart. This organ, already affected by the increased oxygen demand due to the virus infection and with an increased myocardial load induced by the high metabolic rate, enters into a greater imbalance between myocardial oxygen supply and demand [22].

All disturbances caused in the respiratory system can lead to pneumonia or severe acute respiratory syndrome (SARS). SARS is a lung disease that prevents sufficient oxygen from reaching the lungs and circulation. Once this point of the disease is reached, the virus reaches the bloodstream, establishing viremia, and the systemic character of the disease [23]. In consequence, the extrapulmonary manifestations begin in sites whose tissues express the ACE2 receptor [17, 19]. Regarding the heart, approximately $7.5 \%$ of cardiac cells express the ACE2 receptor, which makes it a potentially affected organ in cases of infection by the new coronavirus $[16,19]$. The mechanisms of SARS-CoV-2 injury are not yet fully elucidated, although they are probably multifactorial [19]. Also, it has not been fully elucidated whether organ and tissue injury in a patient with COVID-19 is a direct or indirect consequence of virus infection [16].

As the disease progresses, the imbalance increases resulting in myocardial injury in COVID-19 patients, especially in patients with cardiovascular disease who have already exhausted the myocardial reserve capacity. Besides, other pathological manifestations ranging from minimal change to interstitial inflammatory infiltration and myocyte necrosis can be found. Micro-thrombosis, vascular inflammation, shock, and arrhythmia can also be found [24]. The excessive inflammatory response, called "cytokine storm", induced by the viral infection can generate a hypercoagulation due to the inflammation induced by the vascular system, instability of atheroma plaques and pathological activation of thrombin $[16,22,24]$. This hypercoagulation together with the uncontrolled macrophage response and pathological activation of thrombin are associated with the events of peripheral ischemia, deep vein thrombosis (DVT), pulmonary thromboembolism (PTE), and disseminated intravascular coagulation (DIC) [2, 22].

The rampant production of cytokines can indiscriminately injure many vital organs. In the heart, the injury to the myocardium promotes an increase in the levels of myocardial biomarkers, such as troponin. Other changes can also be observed in the electrocardiogram (ECG) and echocardiogram (ECO) [16]. Another important biomarker in the infection by SARS-CoV-2 is the D-dimer. This biomarker is indicative of a state of hypercoagulation and the risk of SARS. It is associated with an increased risk of mortality $[2,16,22]$. In the case of pediatric patients, an exaggerated response to the virus is extremely important. However, in Italy, the United Kingdom, Spain, the United States, France, and Switzerland, there have been reports of children 
presenting with a multisystemic inflammatory syndrome, a rare but very serious condition. In addition, it was found the children presented with high fever and a varying number of symptoms previously associated with Kawasaki disease, such as conjunctivitis, lymphadenopathy, mucocutaneous rash, and coronary artery dilation, and, in the most severe cases, cardiovascular shock, encephalitis, and multiple organ failure. Kawasaki disease is a vasculitis that affects mediumsized arteries, with a higher incidence in children under 5 years of age and is the main cause of acquired heart disease in developed countries where streptococcal infections are commonly treated, and rheumatic fever is rare [25, 26].

In this present study, two types of drugs with potential use in the treatment of COVID-19 were addressed: glucocorticoids and azithromycin.

\section{Glucocorticoids}

Corticosteroids have been widely used during the outbreaks of severe acute respiratory syndrome (SARS) and the Middle East respiratory syndrome (MERS) and are being used in patients with SARS-CoV-2 in addition to other types of drugs. This medicine is already used in many indications for its powerful anti-inflammatory and immunosuppressive effects [24, 27, 28]. Corticosteroids are currently among the main drugs used in the treatment of COVID-19 as they decrease the effect of inflammation-promoting substances such as cytokines $[2,3]$. It is suggested that the effects of corticoids vary depending on the stage of the disease. For example, low doses of corticosteroids during the pulmonary phase could be beneficial in preventing hyper inflammation, while higher doses would be needed to decrease an already existing hyperinflammatory process [29].

In SARS-CoV infection, as in influenza, inflammation is associated with adverse outcomes. Corticosteroids suppress lung inflammation but can also inhibit immune responses and pathogen clearance if used early. In COVID-19, inflammation persists after viral clearance. Pulmonary histology performed in cases of SARS and MERS infections reveal inflammation and diffuse alveolar damage, with a report suggestive of hemophagocytosis [30]. However, a systematic review and meta-analysis from ten observational studies on influenza with 6548 patients showed increased mortality in patients receiving corticosteroids [31].

In March 2020, the RECOVERY (Randomized Evaluation of COVID-19 Therapy) trial was established as a randomized clinical trial to test a range of potential treatments for the disease, including low-dose dexamethasone. Over 11,500 patients were enrolled in the UK. The scientists concluded that dexamethasone decreases the risk of death by $35 \%$ in patients who were receiving mechanical ventilation because of COVID-19 [32]. Based on these results, one death would be prevented with this treatment in every eight patients on mechanical ventilation or one in every 25 among those who only required oxygen. In patients with mild cases of coronavirus, there was no difference in mortality. Data from Brazilian survey also indicate that the medication represents an improvement for patients with COVID-19 who require ventilatory support. The survival benefit is significant in patients sick enough to need oxygen, for whom dexamethasone should now become the basic treatment [33].

In September 2020, the WHO published a guideline recommending the use of corticosteroids in mild cases of COVID-19. Corticosteroids (dexamethasone and prednisolone) are on the WHO model list of essential medicines. The medicines on this list are those that should be a priority for governments in terms of supply and should be available at a low cost to stakeholders. The guideline suggests not using corticosteroids in mild cases of the disease [3].

Each case should be evaluated individually by the attending physician; there are many variations and different responses from one patient to another. However, results show that the benefit of corticosteroids concerning mortality is of critical importance for patients with little or no treatment variation in the severe case of the disease [34].

\section{Azithromycin}

Azithromycin is one of the safest and well-tolerated antibiotics and has a broad antimicrobial spectrum. Recent work demonstrates that macrolides such as erythromycin have anti-rhinoviral activity in bronchial epithelial cells in addition to increasing the expression of interferon-stimulated genes [35-37]. Several macrolide antibiotics have anti-inflammatory effects by inhibiting the production of pro-inflammatory cytokines in vitro. This effect occurs through suppression of NF- $\kappa \mathrm{B}$ activation. The reduction of cytokines, such as IL-6 and IL-8, may be an advantageous mechanism leading to attenuation of airway inflammation [38]. The fact that COVID-19 patients present complications of pneumonia and acute respiratory distress is a justification for the therapeutic use of azithromycin for the current pandemic [39].

In Brazil, the Ministry of Health booklet guides the use of azithromycin in the first 5 days of treatment of the disease [40]. Even without clear evidence, most countries used hydroxychloroquine combined or not with azithromycin making it one of the combinations used in studies related to coronavirus [41, 42].

Conflicting results concern the positive effect of hydroxychloroquine in the treatment of COVID-19. Hydroxychloroquine could block the virus from entering the cell by interfering with the glycosylation of ACE2 receptors. The therapeutic action of hydroxychloroquine could be potentiated 
by azithromycin, with the ability to reduce the activities of IL6 and TNF- $\alpha$ [43]. Although the antibiotic has no proven efficacy against the virus, if consented by the patient it can be used off label [41, 42, 44].

The major concern related to the use of these two drugs is due to cardiac toxicity when used alone and, of course, in combination. Million et al. conducted an analysis of 1061 patients with a positive test for SARS-CoV-2 treated for at least 3 days with a combination of hydroxychloroquine and azithromycin [45]. Among the eight $(0.75 \%)$ patients who died, all deaths resulted from respiratory failure and not from cardiac toxicity. Rosenberg et al. produced a cohort study in 25 New York hospitals, where they randomly chose 1438 patients out of 7914 diagnosed with COVID-19 and noted that patients with a greater chance of cardiac arrest were using hydroxychloroquine and azithromycin in comparison with patients who did not use the drugs [39].

Furtado et al. evaluated whether the inclusion of azithromycin to the standard treatment, where hydroxychloroquine is included, could contribute to a better outcome of critically ill hospitalized patients with COVID-19 [41]. As a result, they noted no improvement with the addition of the drug. The combination of azithromycin and hydroxychloroquine was also addressed by Rosenberg et al. [39]; Molina et al. [40] and Hraiech et al. [41]. None of the aforementioned authors cited any benefit associated with the two drugs in terms of reduced mortality or viral elimination. In these studies, there were no significant differences between the groups with or without the use of the drugs. The use of azithromycin would be appropriate only for the treatment of bacterial co-infections related to the disease. For example, in some scenarios of SARS-CoV-2 infection, the presence of pus and fluid in the lung alveoli creates a nutritive environment for bacteria such as Pseudomonas aeruginosa and Staphylococcus aureus [46]. The studies show the action of azithromycin alone makes it even more difficult to demonstrate that the role of the drug as a therapeutic agent in COVID-19.

The failure of the azithromycin and hydroxychloroquine combination led to the non-inclusion of azithromycin in the Solidarity study conducted by WHO [39]. Azithromycin is currently one of the most widely prescribed for COVID-19 patients, however, in most of the studies cited in this review, it showed no effect in combating the virus. In addition, the mass distribution of azithromycin may propagate macrolide resistance [47] and the WHO no longer recommends the use of antibiotics in the treatment of COVID-19, except in those patients with bacterial infection secondary to the disease.

\section{Conclusion}

We conclude that corticosteroids may be recommended in patients who are in a severe condition since the use of dexamethasone lowered mortality in COVID-19 patients receiving mechanical ventilation or oxygen therapy. Among patients who did not require oxygen therapy, corticosteroids increased mortality [32].

There is no evidence of beneficial effects on the risk of progression to mechanical ventilation or length of the hospital for patients in the use of azithromycin. Several large-scale randomized clinical trials found no benefit of azithromycin in patients hospitalized with COVID-19 [34].

\section{References}

1. Pimentel RMM, Daboin BEG, de Oliveira AG, Macedo H. The dissemination of COVID-19: an expectant and preventive role in global health. J Hum Growth Dev. 2020;30:135-40. https://doi. org/10.7322/JHGD.V30.9976.

2. Kakodkar P, Kaka N, Baig M. A comprehensive literature review on the clinical presentation, and management of the pandemic coronavirus disease 2019 (COVID-19). Cureus. 2020;12(4):e7560. https://doi.org/10.7759/cureus.7560.

3. Xu X, Ong YK, Wang DY. Role of adjunctive treatment strategies in COVID-19 and a review of international and national clinical guidelines. Mil Med Res. 2020;7(1):22. https://doi.org/10.1186/ s40779-020-00251-x.

4. Fu Y, Cheng Y, Wu Y. Understanding SARS-CoV-2-mediated inflammatory responses: from mechanisms to potential therapeutic tools. Virol Sin. 2020;35(3):266-71. https://doi.org/10.1007/ s12250-020-00207-4.

5. Malik YA. Properties of coronavirus and SARS-CoV-2. Malays J Pathol. 2020;42(1):3-11.

6. Chen T, Wu D, Chen H, Yan W, Yang D, Chen G, et al. Clinical characteristics of 113 deceased patients with coronavirus disease 2019: retrospective study. BMJ. 2020;368:m1091. https://doi.org/ 10.1136/bmj.m1091.

7. Jordan RE, Adab P, Cheng KK. COVID-19: risk factors for severe disease and death. BMJ. 2020;368:m1198. https://doi.org/10. 1136/bmj.m1198.

8. Javanmard SH, Heshmat-Ghahdarijani K, Vaseghi G. Angiotensinconverting-enzyme inhibitors (ACE inhibitors) and angiotensin II receptor blocker (ARB) use in COVID-19 prevention or treatment: a paradox. Infect Control Hosp Epidemiol. 2021;42:118-9. https:// doi.org/10.1017/ice.2020.195.

9. Jin Y, Yang H, Ji W, Wu W, Chen S, Zhang W, et al. Virology, epidemiology, pathogenesis, and control of COVID-19. Viruses. 2020;12(4):372. https://doi.org/10.3390/v12040372.

10. Kang Y, Chen T, Mui D, Ferrari V, Jagasia D, Scherrer-Crosbie M, et al. Cardiovascular manifestations and treatment considerations in COVID-19. Heart. 2020;106:1132-41. https://doi.org/10.1136/ heartjnl-2020-317056.

11. Schulte W, Bernhagen J, Bucala R. Cytokines in sepsis: potent immunoregulators and potential therapeutic targets - an updated view. Mediators Inflamm. 2013;2013:165974. https://doi.org/10. 1155/2013/165974.

12. Lin L, Lu L, Cao W, Li T. Hypothesis for potential pathogenesis of SARS-CoV-2 infection-a review of immune changes in patients 
with viral pneumonia. Emerg Microbes Infect. 2020;9:727-32. https://doi.org/10.1080/22221751.2020.1746199.

13. Arabi YM, Mandourah Y, Al-Hameed F, Sindi AA, Almekhlafi GA, Hussein MA, et al. Corticosteroid therapy for critically ill patients with middle east respiratory syndrome. Am J Respir Crit Care Med. 2018;197:757-67. https://doi.org/10.1164/rccm. 201706-1172OC.

14. World Health Organization (2020) Clinical management of severe acute respiratory infection when novel coronavirus (2019-nCoV) infection is suspected: interim guidance, 28 January 2020 . WHO. Retrieved from WHO/2019-nCoV/clinical/2020.5. https://apps. who.int/iris/handle/10665/330893

15. González FEJ, Alulima GDFJ, Auquilla ELS, Sanclemente JCM, Vasquez PAM, Páez JLV. Hypercoagulability, intravascular thrombosis, and thrombocytosis associated with COVID-19. Case report. Rev Bionatura. 2020;5:1138-41. https://doi.org/10.21931/ $\mathrm{RB} / 2020.05 .02 .11$.

16. Huang C, Wang Y, Li X, Ren L, Zhao J, Hu Y, et al. Clinical features of patients infected with 2019 novel coronavirus in Wuhan, China. Lancet. 2020;395:497-506. https://doi.org/10.1016/S01406736(20)30183-5.

17. Rowley AH. Understanding SARS-CoV-2-related multisystem inflammatory syndrome in children. Nat Rev Immunol. 2020;20:453-4. https://doi.org/10.1038/s41577-020-0367-5.

18. Consiglio CR, Cotugno N, Sardh F, Pou C, Amodio D, Rodriguez $\mathrm{L}$, et al. The immunology of multisystem inflammatory syndrome in children with COVID-19. Cell. 2020;183:968-981.e7. https:// doi.org/10.1016/j.cell.2020.09.016.

19. Verdecchia P, Cavallini C, Spanevello A, Angeli F. The pivotal link between ACE2 deficiency and SARS-CoV-2 infection. Eur J Int Med. 2020;76:14-20. https://doi.org/10.1016/j.ejim.2020.04. 037.

20. Yildiz H, Bailly S, Van Den Neste E, Yombi JC. Clinical management of relapsed/refractory hemophagocytic lymphohistiocytosis in adult patients: a review of current strategies and emerging therapies. Ther Clin Risk Manag. 2021;17:293-304. https://doi. org/10.2147/tcrm.s195538.

21. Li N, Jie Z. The application of corticosteroids in COVID-19: a two-edged sword. J Transl Int Med. 2020;8:66-70. https://doi.org/ 10.2478/jtim-2020-0011.

22. Russell CD, Millar JE, Baillie JK. Clinical evidence does not support corticosteroid treatment for 2019-nCoV lung injury. Lancet. 2020;395:473-5. https://doi.org/10.1016/S0140-6736(20) 30317-2.

23. Ni YN, Chen G, Sun J, Liang BM, Liang ZA. The effect of corticosteroids on mortality of patients with influenza pneumonia: a systematic review and meta-analysis. Crit Care. 2019;23(1):99. https://doi.org/10.1186/s13054-019-2395-8.

24. University of Oxford. Low-cost dexamethasone reduces death by up to one third in hospitalised patients with severe respiratory complications of COVID-19 - the RECOVERY trial. Oxford University news release. University of Oxford; 2020.

25. Bakheit AH, Al-Hadiya BM, Abd-Elgalil A. Azithromycin. Profiles Drug Subst Excip Relat Methodol. 2014;39:1-40.

26. Takizawa H, Desaki M, Ohtoshi T, Kawasaki S, Kohyama T, Sato $\mathrm{M}$, et al. Erythromycin modulates IL-8 expression in normal and inflamed human bronchial epithelial cells. Am J Respir Crit Care Med. 1997;156:266-71. https://doi.org/10.1164/ajrccm.156.1. 9612065.

27. Suzuki T, Yamaya M, Sekizawa K, Hosoda M, Yamada N, Ishizuka S, et al. Erythromycin inhibits rhinovirus infection in cultured human tracheal epithelial cells. Am J Respir Crit Care Med. 2002;165:1113-8. https://doi.org/10.1164/ajrccm.165.8.2103094.

28. Khair OA, Devalia JL, Abdelaziz MM, Sapsford RJ, Davies RJ. Effect of erythromycin on Haemophilus influenzae endotoxin-induced release of IL-6, IL- 8 and sICAM-1 by cultured human bronchial epithelial cells. Eur Respir J. 1995;8:1451-7.

29. Desaki M, Takizawa H, Ohtoshi T, Kasama T, Kobayashi K, Sunazuka T, et al. Erythromycin suppresses nuclear factor- $\kappa \mathrm{B}$ and activator protein-1 activation in human bronchial epithelial cells. Biochem Biophys Res Commun. 2000;267:124-8. https:// doi.org/10.1006/bbrc.1999.1917.

30. Russell CD, Millar JE, Baillie JK. Clinical evidence does not support corticosteroid treatment for 2019-nCoV lung injury. Lancet. 2020;395(10223):473-5. https://doi.org/10.1016/S0140-6736(20) 30317-2.

31. Geng YJ, Wei ZY, Qian HY, Huang J, Lodato R, Castriotta RJ. Pathophysiological characteristics and therapeutic approaches for pulmonary injury and cardiovascular complications of coronavirus disease 2019. Cardiovasc Pathol. 2020;47:107228. https://doi. org/10.1016/j.carpath.2020.107228.

32. Pasin L, Navalesi P, Zangrillo A, Kuzovlev A, Likhvantsev V, Hajjar LA, et al. Corticosteroids for patients with coronavirus disease 2019 (COVID-19) with different disease severity: a metaanalysis of randomized clinical trials. J Cardiothorac Vasc Anesth. 2021;35:578-84. https://doi.org/10.1053/j.jvca.2020.11.057.

33. Tomazini BM, Maia IS, Cavalcanti AB, Berwanger O, Rosa RG, Veiga VC, et al. Effect of dexamethasone on days alive and ventilator-free in patients with moderate or severe acute respiratory distress syndrome and COVID-19: the CoDEX randomized clinical trial. JAMA. 2020;324(13):1307-16. https://doi.org/10.1001/ jama.2020.17021.

34. Furtado RHM, Berwanger O, Fonseca HA, Corrêa TD, Ferraz LR, Lapa MG, et al. Azithromycin in addition to standard of care versus standard of care alone in the treatment of patients admitted to the hospital with severe COVID-19 in Brazil (COALITION II): a randomised clinical trial. Lancet. 2020;396:959-67. https://doi. org/10.1016/S0140-6736(20)31862-6.

35. Oldenburg CE, Doan T. Azithromycin for severe COVID-19. Lancet. 2020;396:936-7. https://doi.org/10.1016/S0140-6736(20) 31863-8.

36. Desaki M, Okazaki H, Sunazuka T, Omura S, Yamamoto K, Takizawa $\mathrm{H}$. Molecular mechanisms of anti-inflammatory action of erythromycin in human bronchial epithelial cells: possible role in the signaling pathway that regulates nuclear factor- $\mathrm{\kappa B}$ activation. Antimicrob Agents Chemother. 2004;48:1581-5. https://doi.org/ 10.1128/AAC.48.5.1581-1585.2004.

37. Bolay H, Gül A, Baykan B. COVID-19 is a real headache! Headache. 2020;60:1415-21. https://doi.org/10.1111/head.13856.

38. Sultana J, Cutroneo PM, Crisafulli S, Puglisi G, Caramori G, Trifirò G. Azithromycin in COVID-19 patients: pharmacological mechanism, clinical evidence and prescribing guidelines. Drug Saf. 2020;43:691-8. https://doi.org/10.1007/s40264-020-00976-7.

39. Rosenberg ES, Dufort EM, Udo T, Wilberschied LA, Kumar J, Tesoriero J, et al. Association of treatment with hydroxychloroquine or azithromycin with in-hospital mortality in patients with COVID-19 in New York State. JAMA. 2020;323:2493-502. https://doi.org/10.1001/jama.2020.8630.

40. Molina JM, Delaugerre C, Le Goff J, Mela-Lima B, Ponscarme $\mathrm{D}$, Goldwirt L, et al. No evidence of rapid antiviral clearance or clinical benefit with the combination of hydroxychloroquine and azithromycin in patients with severe COVID-19 infection. Med Mal Infect. 2020;50(4):384. https://doi.org/10.1016/j.medmal. 2020.03.006.

41. Hraiech S, Bourenne J, Kuteifan K, Helms J, Carvelli J, Gainnier $\mathrm{M}$, et al. Lack of viral clearance by the combination of hydroxychloroquine and azithromycin or lopinavir and ritonavir in SARS-CoV-2-related acute respiratory distress syndrome. Ann Intensive Care. 2020;10(1):63. https://doi.org/10.1186/ s13613-020-00678-4. 
42. Firth A, Prathapan P. Azithromycin: the first broad-spectrum therapeutic. Eur J Med Chem. 2020;207:112739. https://doi.org/ 10.1016/j.ejmech.2020.112739.

43. Lucien MAB, Canarie MF, Kilgore PE, Jean-Denis G, Fénélon $\mathrm{N}$, Pierre M, et al. Antibiotics and antimicrobial resistance in the COVID-19 era: perspective from resource-limited settings. Int J Infect Dis. 2021;104:250-4. https://doi.org/10.1016/j.ijid.2020. 12.087 .

44. Doan T, Worden L, Hinterwirth A, Arzika AM, Maliki R, Abdou $\mathrm{A}$, et al. Macrolide and nonmacrolide resistance with mass azithromycin distribution. N Engl J Med. 2020;383:1941-50. https://doi.org/10.1056/nejmoa2002606.

45. Million M, Lagier JC, Gautret P, Colson P, Fournier PE, Amrane S, et al. Early treatment of COVID-19 patients with hydroxychloroquine and azithromycin: a retrospective analysis of 1061 cases in Marseille, France. Travel Med Infect Dis. 2020;35:101738. https://doi.org/10.1016/j.tmaid.2020.101738.

46. Kim D, Lee JY, Yang JS, Kim JW, Kim VN, Chang H. The architecture of SARS-CoV-2 transcriptome. Cell. 2020;181:914-921. e10. https://doi.org/10.1016/j.cell.2020.04.011.

47. World Health Organization. Corticosteroids for COVID-19: living guidance. World Health Organization; 2020. p. 1-25.

Publisher's Note Springer Nature remains neutral with regard to jurisdictional claims in published maps and institutional affiliations. 